Proceedings of the 1996 IEEE

International Conference on Robotics and Automation

Minneapolis, Minnesota - April 1996

\title{
Mechanism Design of A New Multifingered Robot Hand
}

\author{
Li-Ren Lin * and Han-Pang Huang * * \\ Robotics Laboratory, Department of Mechanical Engineering \\ National Taiwan University, Taipei, TAIWAN 10674, R.O.C. \\ TEL/FAX: (886)2-3633875 \\ e-mail: hphuang@w3.me.ntu.edu.tw \\ ** Professor and correspondence addressee \\ * Graduate student
}

\begin{abstract}
A new five-finger robot hand (NTU hand) with seventeen degrees of freedom (DOF) is developed in this paper. In contrast to traditional tendon-driven robot, the NTU hand has an uncoupled configuration that each finger and joint are all individually driven. While all actuators, mechanical parts and sensors are packed on the hand, the size of NTU hand is almost the same as a human hand. Such compact design makes the hand easily adapt to the industrial robot arm and the prosthetic applications. According to the mechanical structure of the NTU hand, the direct and inverse kinematics are also developed in this paper.
\end{abstract}

\section{Introduction}

The operation of multifingered robot hands for fine motion and dexterous manipulations is an interesting topic in the research and applications of robotics. The multifingered robot act as a multipurpose gripping device for various tasks. Since it is designed to substitute some work of human hands, most multifingered robots duplicate the shape and function of human hands. In order to manipulate various objects and tools, dexterity is the first requirement for the multifingered robots. In addition to the dexterous manipulation, the ability to perform power grasp is also required. The size of the hand is a significant part in the research. A compact enough multifingered robot can be directly attached to the end of an industrial robot arm, or play a role in the prosthetic applications.

Many multifingered robot hands have been developed $[4,8,10,11]$. The number of fingers ranges from three to five. An example of three-fingered robot is the JPL/Stanford hand [9]. Each finger has three DOFs and is driven by four motors through tendon cables, two parallel axis joints provide rotation and the third proximal joint, perpendicular to the other joints, provides the sideward motion. Due to large number of motors and strong coupling in the tendon configuration, the control system of JPL/Stanford hand is very complicated. In addition, it is also difficult to maintain calibration using the tension of four cables in a finger.
The Utah/MIT hand [4] has one thumb and three fingers. Each finger has four joints. Three parallel joints provide the rotation and the proximal joint supplies the lateral action. Due to four DOFs in each finger, eight independent tendons and pneumatic cylinders are required. Each pair of tendons must keep tension to maintain the joint of the robot finger. Once a lateral motion is performed, one joint is activated and the other three joints keep stationary so that tension on eight tendons must be re-calculated. The coupling problem also causes the Utah/MIT hand to use large number of actuators and complex control system [7]. The above two fingered hands are bulky because of the tendon driven configurations and associated control systems.

The Belgrade/USC hand [10] has five fingers and four motors, two motors for the thumb and two for the other fingers. Each finger has three parallel axis joints with the adaptability feature and one DOF. The hand only provides simple grasping capacity rather than dexterous manipulation. The multifingered robot of Yaskawa electric corporation also has three finger and nine DOFs [11]. This hand just can utilize the fingertip because of the bulky cylinder finger segment.

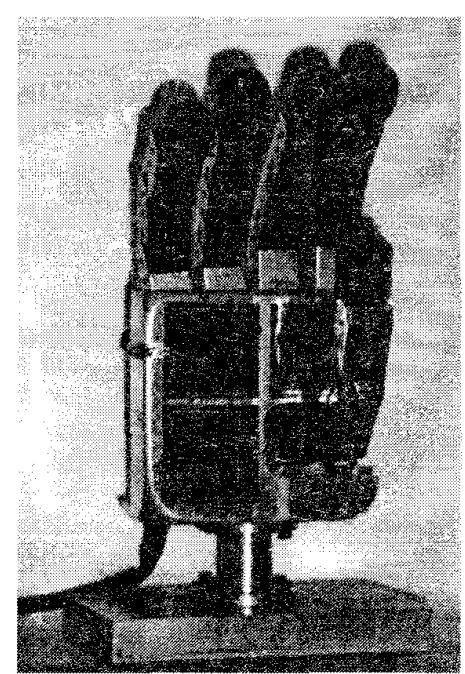

Figure 1. The NTU hand 
This paper is organized into two parts. The first part is the mechanism design of the NTU hand, as shown in Fig.1. This part presents the design concept of the NTU hand and shows the detailed scheme of mechanism. Due to the uncoupled mechanism, each finger and joints are all individually driven. Hence, the dexterity of the NTU hand can be obtained from the uncoupled arrangement. The specifications and some issues of the mechanical consideration are also stated. The second part is the kinematics of the NTU hand. The direct kinematics, inverse kinematics of fingers are presented.

\section{Mechanism design}

Many multifingered hands are driven by tendon cables. One reason is that the cables act as the muscles of the human hand. Another reason is that the actuators, reduction gears and sensors can be remotely installed to keep the hand itself not so huge. However, all traditional hands suffer from bulky mechanism and are inconvenient for practical applications. In this paper, we intend to design a multifingered robot hand with an uncoupled driven mechanism with modular design, and to limit the hand size for both industrial and prosthetic applications.

Our goals of the mechanism design are aimed to several considerations. The first one aims to the functionality purpose; i.e., the number of finger and DOF. It is known that three hard fingers are required for a force closure grasp of a 2-D object, and four fingers are required to grasp a 3-D object [5]. The realistic model of fingertip can reduce one finger number required in the same conditions [6]. However, finger number and DOF of the artificial hand are preferred to the human hand. The second goal is the size. We wish the size of the hand including the overall driven mechanism is the same as the human hand. Limitation of the size makes the hand suitable for robotics and rehabilitation application. Once all parts are packed in the hand itself, the hand can be easily attached to the wrist of industrial robot or the casualty.

The next goal focused on the fabrication and maintenance. If the same parts are repeatedly used in the modular design of hand, the number of type of parts will be reduced and the cost will be down. Once the fingers are independent and exchangeable, the maintenance simply replace the damaged finger assembly without recalibration of whole system. The last goal deals with the potential of improvement. The performance can be enhanced by replacing the parts whenever better materials are available. But, the main design is preserved. Once the materials of transmission or the power of actuator are improved, the performance of the hand is also enhanced. For different applications, we just change the kind of the material without changing the main design; e.g., the steel material for robotics application and the composite material for rehabilitation.

Based on the above goals, the design of the NTU hand is established. The NTU hand has five fingers with seventeen DOFs. Both thumb and the first finger have four joints; two at knuckle, one between proximal and middle finger segments, and one between middle and distal finger segments. Other fingers have three joints; but only one at knuckle. Each finger is equipped with tactile sensors to detect grasping force. Due to cost issue and practical implementation, the tactile sensors are attached to the inner sides of finger segments and the palm, as shown in Fig.1. We will present the concept and details of design in the subsequent sections.

\subsection{Hand mechanism}

The idea of design is obtained from the human hand. Since the structure of the fingers of human hand are almost the same and independent, as shown in Fig.2, this feature gives us the idea to design the hand beginning with finger and to simplify the development. The human finger consists of finger segments This fact also gives us the inspiration to design an independently driven finger segment to construct a whole finger. The auxiliary devices for the artificial finger are also required for the lateral motion, as the function of muscles in the palm.

\subsubsection{The finger and the finger segment}

The finger of the NTU hand consists of distal segment, middle segment, proximal segment and base finger segment. To ease the manufacture and assembly, the group technology is applied to the design of the fingers and finger segments. The design should avoid the coupling problem of tendon driven structure and limit the size to make it easy for applications. Once an individual finger is constructed, the mechanism of whole robot hand is almost complete. In order to meet the requirement of the independently driven, it is essential to design a finger with all equipped parts.

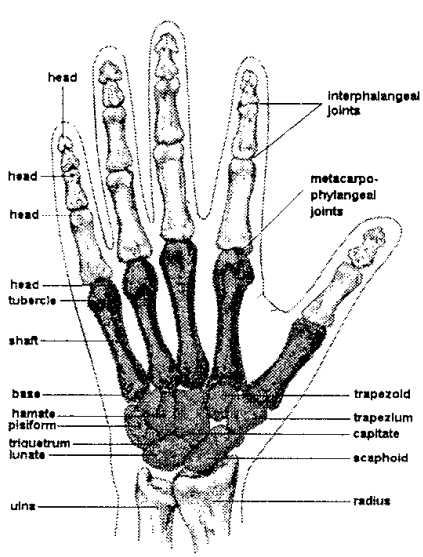

Figure 2. The human hand [2] 


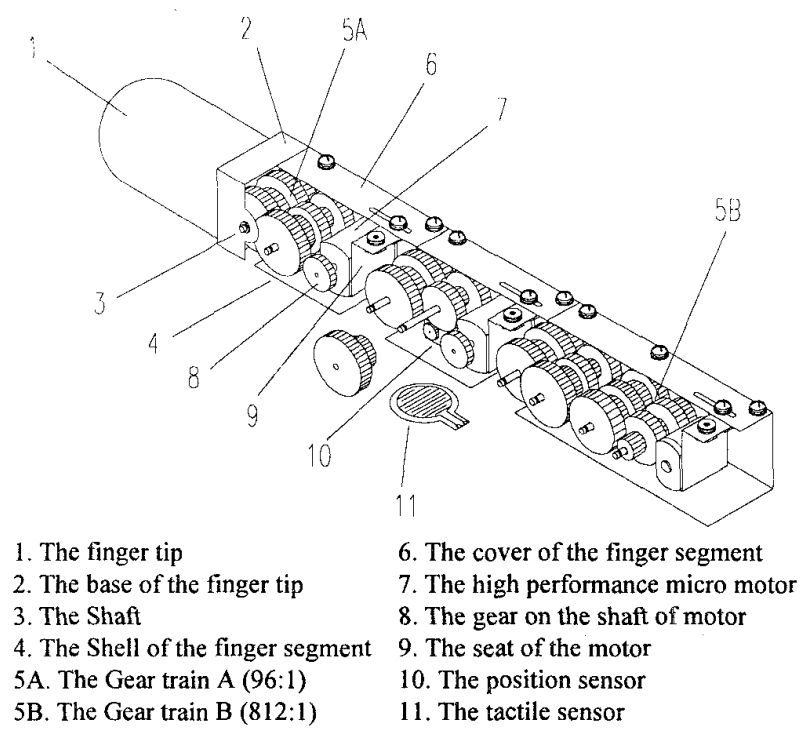

Figure 3 . The assembly of the fundamental finger

The design of finger is shown in Fig.3. Each finger segment, except the distal segment, contains one high performance micro motor that drives a set of specially arranged gear trains to rotate the previous finger segment, as shown in Fig.4. The gear ratio of the middle and proximal finger segments is about one hundred, but the gear ratio of the base segment is about one thousand for the sake of heavy load. The position sensor of each joint is also installed in each finger segment. It is driven by the gear within the gear trains, and is proportional to the angle of the finger joint.

There are many electrical wires in the inner space of each segment shell, the placement of sensors and motors must be taken into account during the assembly process. Since the position sensor is driven by gear within the gear trains, its calibration must be accomplished during the assembly process.

\subsubsection{The auxiliary device for the lateral joints}

To ease the manufacture effort, the lateral rotation of the thumb and the first finger is achieved by adding auxiliary device to a fundamental finger, and the mechanic power is provided by an additional finger segment. The overall design scheme of the NTU hand is shown in the Fig.5. In the design of the lateral joint of the thumb, we attach a large gear on the thumb and use a modified finger segment to drive it. In the design of the lateral joint of the first finger, we use an adjustable linkage with a ball joint to transport the mechanic power, they convert the rotation of the gear into the linear motion. The thumb and the first finger, each with four DOFs provide approximately anthropomorphic motions similar to the natural human hand.

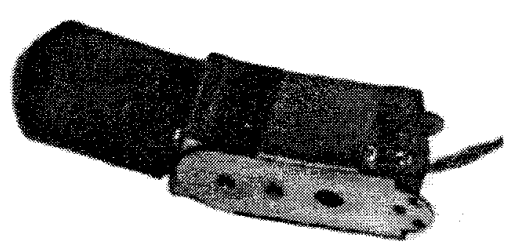

Figure 4. The assemble of the distal segment and middle segment (A set of gear train, micro motor and a potential meter for the detection of joint variable are all packed in the middle segment)

\subsubsection{The paim and the wrist}

The palm serves as a structural mounting base for the thumb, fingers and the wrist. The last three fingers are located on the palm by the fasten plane that arranges the fingers to cooperate with the thumb and the first finger. The upper space of the palm provides the location to install the controller and various electronic components [3]. The wrist shown in the Fig.5 is designed to connect the NTU hand to the robot arm PUMA 560. Once the design of the NTU hand is modified for rehabilitation, the wrist needs to be changed to adapt the casualty.

\subsection{Problems of the present design}

The current design suffers from some problems. These problems determine the performance of the NTU hand. This sub-section states what problems encountered in the present design and how we solve.

\subsubsection{Problem of the gear driven}

The driven mechanism of the NTU hand is performed by a set of gear trains. The disadvantage of the gear driven is the backlash problem. It means the accuracy of the joint position will not be good. The problem is not serious while the hand operates to grasp object. The backlash will not happen because the gears are keeping contact during the grasp operation. The most important is the tactile sensing while grasping.

The sensing of the NTU hand is divided into two parts, one is the joint variables, the other is the tactile sensing. According to the grasp of the human, the posture of the hand is not very accurate because the main goal of grasp is to manipulate an object. It means the function of the finger movement is keeping contact with the object. The design of the NTU hand also follows the guide line that the tactile sensing is the first consideration and then movement of the finger joint. It also implies that we can reduce the cost in the hand design. 


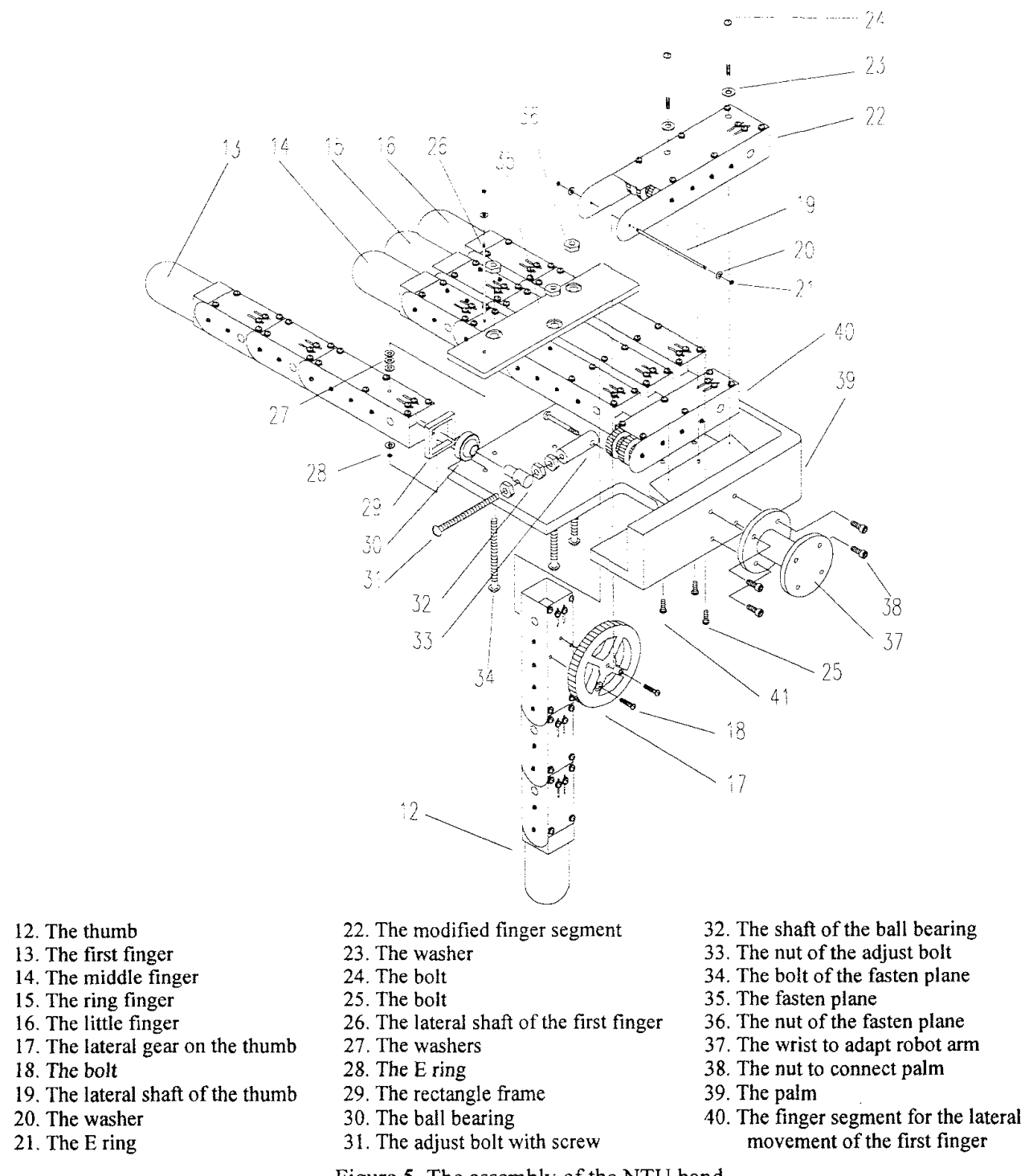

Figure 5. The assembly of the NTU hand

\subsubsection{Force, torque and heat $\operatorname{sink}$}

The torque output of the finger segment is high because of the high gear ratio. Hence, the force of the hand will be large. The limitation of the force output is determined by the capacity of motor and the strength of the gears. The modulus and thickness of the final stage of the gear trains should be larger than the pervious stage to stand the larger torque, or use the high strength of gear in the final stage of the gear trains.

The heat sink of the motor is also related the force output. The torque of the motor decreases while the heat accumulates in the chamber of the finger segment. In the prototype of the NTU hand, we use a seat of motor to transfer the heat to the outside of the finger segment.

\subsubsection{Material and the applications}

The selection of the material depends on the application of the hand. The prototype of the NTU hand, as shown in Fig.1, is almost built by the steel and aluminum except the polymer finger tip. The weight of the hand, which is appropriate for the robotics application, is listed in the Table 1. Due to the compact size of the whole system, the high possibility for prosthetic application is one advantage of the NTU hand. For the prosthetic application, the material can be chosen to reduce the weight without decreasing the strength. It may be some polymer or composite material. The material of the hand can be chosen in terms of different applications, but the design spirit of the hand will not change, or just slight modifications. 


\subsection{Specifications of the prototype}

Since the fundamental finger of the NTU hand has three joints, numbered from 1 to 3 , the specifications of the three joints of each finger are all the same. The lateral joints (joint 0 ) of the thumb and the first finger are performed by different auxiliary devices. The specifications of these two joints are special.

The specifications of the NTU hand are listed in Table 1. The prototype of the NTU hand is made of metal. Its weight can be reduced when other materials are used. The next prototype of the NTU hand will use some light material to reduce its weight. The rated weight of the object to be manipulated is determined by dexterous operation while the object is operated by fingertip of the hand. The rated weight of object to be grasped is determined by power grasp operation while the object is grasped by fingertip and inner links of finger and the palm of the hand.

\section{Kinematics}

\subsection{The direct kinematics}

The coordinate frames of the NTU hand are shown in Fig.6. Note that the thumb and the first finger have four joints, numbered from 0 to 3 ; and the rest of three fingers only have three joints, numbered from 1 to 3 . In the derivation of inverse kinematics for the NTU hand, each finger is treated the same as the thumb or the first finger, except the joint 0 . The link parameters of each finger are listed in Table 2, based on Denavit-Hartenberg method [1]. The matrix ${ }^{j-1} T_{j}^{j}$ is known as the $\mathrm{D}-\mathrm{H}$ transformation matrix for adjacent frames, $j$ and $j-1$. Thus,

\begin{tabular}{|c|c|c|c|c|c|c|}
\hline & Joint $0^{*}$ & Joint $0 !$ & Joint 1 & \multicolumn{2}{|c|}{ Joint 2} & Joint 3 \\
\hline $\begin{array}{c}\max . \text { angular } \\
\text { velocity }(\mathrm{rad} / \mathrm{s})\end{array}$ & 0.21 & 0.39 & 1.05 & \multicolumn{2}{|c|}{5.97} & 7.33 \\
\hline $\begin{array}{l}\text { bandwidth } \\
\text { (Hz) }\end{array}$ & 0.15 & 0.31 & 0.50 & \multicolumn{2}{|c|}{2.85} & 3.50 \\
\hline $\begin{array}{l}\text { torque output } \\
\qquad(\mathrm{g}-\mathrm{cm})\end{array}$ & 3661.9 & 1971.8 & 1350.1 & \multicolumn{2}{|c|}{859.44} & 859.44 \\
\hline \multicolumn{4}{|c|}{ The weight of each finger } & \multicolumn{3}{|c|}{$191.2 \mathrm{~g}$} \\
\hline \multicolumn{5}{|c|}{ The weight of the palm and auxiliary device } & \multicolumn{2}{|c|}{$613.1 \mathrm{~g}$} \\
\hline \multicolumn{5}{|c|}{ The total weight of the NTU hand } & \multicolumn{2}{|c|}{$1569.0 \mathrm{~g}$} \\
\hline \multicolumn{5}{|c|}{ The rated weight of object to be manipulated } & \multicolumn{2}{|c|}{$0.5 \mathrm{Kg}$} \\
\hline \multicolumn{5}{|c|}{ The rated weight of object to be grasped } & \multicolumn{2}{|c|}{$1.0 \mathrm{Kg}$} \\
\hline \multicolumn{5}{|c|}{ The maximum linear velocity of finger tip } & \multicolumn{2}{|c|}{$877.08 \mathrm{~mm} / \mathrm{s}$} \\
\hline \multicolumn{5}{|c|}{ The current consumption of the mechanism } & \multicolumn{2}{|c|}{$0 \mathrm{~A}-8.5 \mathrm{~A}$} \\
\hline
\end{tabular}

Table 1. The specifications of prototype of the NTU hand ( ${ }^{*}$ for thumb, !for first finger)

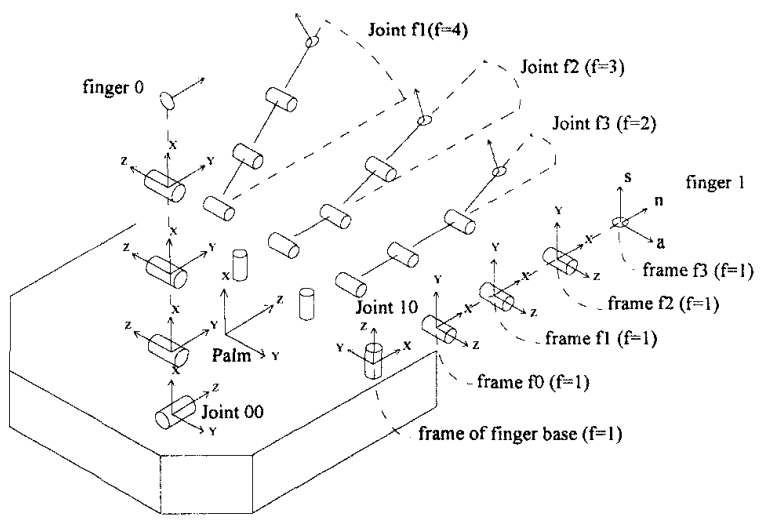

Figure 6. The coordinate frame of the NTU hand

${ }^{j-1} T_{j}^{\prime}=\left[\begin{array}{cccc}\cos \theta_{j} & -\cos \alpha_{j} \sin \theta_{j} & \sin \alpha_{j} \sin \theta_{j} & a_{j} \cos \theta_{j} \\ \sin \theta_{j} & \cos \alpha_{j} \cos \theta_{j} & -\sin \alpha_{j} \cos \theta_{j} & a_{j} \sin \theta_{j} \\ 0 & \sin \alpha_{j} & \cos \alpha_{j} & d_{j} \\ 0 & 0 & 0 & 1\end{array}\right]$

The homogeneous matrix ${ }^{\text {base }} T_{j}^{f}$ which specifics the location of the $j$-th coordinate frame of the $f-t h$ finger with respect to the finger base of the coordinate system, is the chain product of successive ${ }^{j-1} T_{j}^{j}$, and is expressed as

${ }^{\text {base }} T_{3}^{f}={ }^{\text {base }} T_{0}^{f 0} T_{1}^{f}{ }^{1} T_{2}^{f}{ }^{2} T_{3}^{f}=\left[\begin{array}{cccc}n_{\text {base }}^{f 3} & s_{\text {base }}^{f 3} & a_{\text {base }}^{f 3} & p_{\text {base }}^{f 3} \\ 0 & 0 & 0 & 1\end{array}\right]$

${ }^{0} T_{3}{ }^{f}={ }^{0} T_{1}^{f}{ }^{1} T_{2}{ }^{f}{ }^{2} T_{3}{ }^{f}=\left[\begin{array}{cccc}n_{0}^{f 3} & s_{0}^{f 3} & a_{0}^{f 3} & p_{0}^{f 3} \\ 0 & 0 & 0 & 1\end{array}\right]$

where $n_{i}^{f \alpha}=\left[\begin{array}{c}n_{i, x}^{f \alpha} \\ n_{i, y}^{f \alpha} \\ n_{i, z}^{f \alpha}\end{array}\right], \quad s_{i}^{f \alpha}=\left[\begin{array}{c}s_{i, x}^{f \alpha} \\ s_{i, y}^{f \alpha} \\ s_{i, z}^{f \alpha}\end{array}\right], \quad a_{i}^{f \alpha}=\left[\begin{array}{l}a_{i, x}^{f \alpha} \\ a_{i, y}^{f \alpha} \\ a_{i, z}^{f \alpha}\end{array}\right]$ and $p_{i}^{f \alpha}=\left[\begin{array}{c}p_{i, x}^{f \alpha} \\ p_{i, y}^{f \alpha} \\ p_{i, z}^{f \alpha}\end{array}\right]$

Upon assigning the joint variables of $f$-th finger, the corresponding transformation matrices bure $T_{3}^{s}$ and " $T_{3}^{\prime}$ can be found. For the configuration of the NTU hand, the matrix ${ }^{0} T_{3}^{j}$ is determined by joints $1,2,3$ for all fingers. Notice that the matrix ${ }^{\text {bar }} T_{3}^{\prime}$ is a function of joint 0 as dealing with the thumb and the first fingers.

The orientation and position of each finger tip can be obtained from Eqns. (2) (3). The normal vector $n_{i}^{\prime 3}$, sliding vector $s_{i}^{r^{3}}$ and approach vector $a_{i}^{r^{3}}$ determine the orientation of the finger tip with respect to the coordinate system $i$. The position vector $p_{i}^{\prime 3}$ represents the spatial shift between the finger tip and the origin of the $i$-th coordinate frame.

\subsection{The inverse kinematics}

The inverse kinematics is derived from a known set of admissible vectors $n_{\text {palm }}^{\prime 3}$ and $p_{\text {patm }}^{\prime 3}$, then is transformed into $n_{b a v e}^{13}$ and $p_{b u s e}^{r 3}$ by homogeneous matrix ${ }^{\text {padm }} T_{\text {bure }}^{r}$. Hence, the $\theta_{0}^{\prime}$ (joint 0 ) of the finger can be obtained from the following equation, 


$$
\theta_{0}^{f}=\operatorname{atan} 2\left(p_{\text {base }, y}^{f 3},\left|p_{\text {base }, x}^{f 3}\right|\right)
$$

where $\operatorname{atan} 2(y, x)$ return $\tan ^{-1}(y / x)$ with proper quadrant.

To match the structure of the NTU hand, the following conditions must be satisfied,

$$
\theta_{0}^{f}=0 \quad \text { for } f=2,3,4
$$

From a known $\theta_{0}^{\prime}$, we can find the corresponding $n_{0}^{\prime 3}$ and $p_{0}^{s 3}$ by homogeneous matrix " $T_{\text {bus }}^{\prime}$. The derivation of the remaining joints is the same for all fingers. The inverse kinematics are shown below. Let

$$
\theta_{s}^{f}=\theta_{1}^{f}+\theta_{2}^{f}+\theta_{3}^{f}=\operatorname{atan} 2\left(n_{0, y}^{f 3}, n_{0, x}^{f 3}\right)
$$

Define $p_{x}^{f}=p_{0, x}^{f 3}-a_{3} \cos \theta_{s}^{f}$ and $p_{y}^{f}=p_{0, y}^{f 3}-a_{3} \sin \theta_{s}^{f}$.

Then, we have

$$
\begin{aligned}
& \theta_{1}^{f}=\operatorname{atan} 2\left(p_{y}^{f}, p_{x}^{f}\right)-\cos ^{-1}\left(\frac{\left(p_{x}^{f}\right)^{2}+\left(p_{y}^{f}\right)^{2}+\left(a_{1}\right)^{2}-\left(a_{2}\right)^{2}}{2 a_{1} \sqrt{\left(p_{x}^{f}\right)^{2}+\left(p_{y}^{f}\right)^{2}}}\right) \\
& \theta_{2}^{f}=\pi-\cos ^{-1}\left(\frac{\left(a_{1}\right)^{2}+\left(a_{2}\right)^{2}-\left(p_{x}^{f}\right)^{2}-\left(p_{y}^{f}\right)^{2}}{2 a_{1} a_{2}}\right)
\end{aligned}
$$

$\theta_{3}^{f}=\theta_{s}^{f}-\theta_{1}^{f}-\theta_{2}^{f}$

If a set of points and corresponding normal vectors are calculated in terms of the above equations, and the solution of joint variables satisfies Table 2 , we claim that there exists an admissible joint solution of a finger for the pair of point and normal vector. Due to the configuration of the NTU hand, the number of solutions generated by the inverse kinematics is only one. It is obvious because the ranges of joint 1,2,3 are limited within 60 degrees [3].

\begin{tabular}{|c|c|c|c|c|c|}
\hline & $\begin{array}{c}\theta_{j} \\
(\text { degree })\end{array}$ & $\begin{array}{c}\alpha_{j} \\
(\text { degree })\end{array}$ & $\begin{array}{c}a_{j} \\
(\mathrm{~mm})\end{array}$ & $\begin{array}{c}d_{j} \\
(\mathrm{~mm})\end{array}$ & $\begin{array}{c}\text { joint range } \\
(\text { degree })\end{array}$ \\
\hline joint $0^{*}, !$ & 0 & 90 & $35.2^{*}, !$ & 0 & $\begin{array}{c}-30 \sim 45^{*} \\
-10 \sim 10 !\end{array}$ \\
\hline joint 1 & 0 & 0 & 34.0 & 0 & $0 \sim 60$ \\
\hline joint 2 & 0 & 0 & 34.0 & 0 & $0 \sim 60$ \\
\hline joint 3 & 0 & 0 & 42.0 & 0 & $0 \sim 60$ \\
\hline
\end{tabular}

Table 2. The link parameters of finger ( ${ }^{*}$ for thumb, 'for first finger)

\section{Conclusions}

The multifingered robot hand, NTU hand, with seventeen degrees of freedom has been designed and fabricated to achieve dexterous manipulation. Since the NTU hand is lighter and much more compact than traditional fingered hands and with an uncoupled driven mechanism, it can be easily attached to the end of a robot arm to perform dexterous manipulation. In particular, it is useful for prosthetic applications. According to the mechanical structure of the NTU hand, the specifications, the direct and inverse kinematics are also presented in this paper.

\section{References}

[1] J. Denavit and R.S. Hartenberg, "A kinematics notation for low-pair mechanism based on matrices," Journal of Applied Mechanics, pp.215221, 1955.

[2] I. Fowler, Human Anatomy, Wadsworth Publishing: Belmont, California, pp.155, 1984

[3] H.P. Huang, L.R. Lin, "Development of A Prosthetic Hand with Multifingers," NSC Report, NSC 83-0422-E002-093.

[4] S.C. Jacobsen, J.E. Wood, D.F. Knutti and K.B. Biggers, "The UTAH/MIT Dextrous Hand: Work in Progress," International Journal of Robotics Research, vol.3, no.4, pp.21-50, 1984.

[5] X. Markenscoff, L. Ni and C.H. Papadimitriou, "The Geometry of Grasping," International Journal of Robotics Research, vol.9, no.1, pp.61-74, 1990.

[6] B. Mirtich and J. Canny, "Easily Computable Optimum Grasps in 2-D and 3-D," IEEE International Conference on Robotics and Automation, pp.739-747, 1994.

[7] T.H. Speeter, "Control of the Utah/MIT Dextrous Hand: Hardware and Software Hierarchy," Journal of Robotics Systems, vol.7, no.5, pp. 759-790, 1990.

[8] T.H. Speeter, "Primitive Based Control of the Utah/MIT Dextrous Hand," IEEE International Conference on Robotics and Automation, pp.886$877,1991$.

[9] S.A. Stansfield, "Knowledge-based Robotic Gasping," IEEE Conference on Robotics and Automation, pp.1270-1275, 1990.

[10] R. Tomovic, G.A. Bekey and W.J. Karplus, "A Strategy for grasp Synthesis with Multifingered Robot Hand," The International Conference on Robotics and Automation, pp.83-89, 1987.

[11] M. Umetsu and K. Oniki, "Compliant Motion Control of Arm-Hand System," JSME International Conference on Advanced Mechatronics, pp.429-432, 1993. 